\title{
Computation of Broadband Noise Radiated by a Ducted Fan in a Uniform Flow
}

\author{
Serge Lewy ${ }^{\dagger}$ \\ Office National d'Etudes et de Recherches Aérospatiales (ONERA), MB 72, 92322 Chatillon, France
}

(Received 30 July 2003; accepted 20 October 2003)

\begin{abstract}
Ducted-fan broadband noise often dominates overall sound levels radiated by a modern aircraft high-bypassratio turbofan at subsonic rotational tip speeds. As a result, its prediction has become a challenge for additional noise reduction. Equations were established in a previous work for a uniform flow inside the duct, the free medium being at rest (case of static tests). However, induct and free-field acoustic powers are not balanced if there is a flow mismatch at the duct exit. Free-field computation is thus extended to a medium in translation which better simulates flight conditions (the same velocity being taken throughout the space). The radiation model of Tyler and Sofrin (Rayleigh's integral) is no longer valid, and a routine based on the Kirchhoff integral equation has been implemented. It is checked that all the previous results are verified, both in the no-flow case and with flow only inside the duct. Computations assuming a uniform flow throughout the space now lead to the same acoustic power inside the duct and in the free field. This is a basic validation of the prediction model. It is mainly found that flow velocity tends to increase rotor forward radiation and to decrease aft radiation.
\end{abstract}

${ }^{\dagger}$ Member of the International Institute of Acoustics and Vibration (IIAV)

\section{Notations}

a $\quad-$ Speed of sound

$A_{m \mu} \quad-$ Modal amplitude of induct acoustic pressure

c $\quad-$ Blade chord

$(D, \theta, \phi)$ - Spherical coordinates in the free field

$D^{\prime}, \hat{d} \quad-$ Modified distances

$F \quad-$ Blade loading

$f \quad-$ Frequency

$\overrightarrow{\mathrm{I}} \quad-$ Acoustic intensity

$\mathrm{J}_{m} \quad-$ Bessel function of the first kind, and of order $m$

$K, k_{t}, k_{z}$ - Total, transverse, and axial wave-numbers

$M_{z} \quad-$ Axial Mach number,

$m, \mu \quad-$ Circumferential and radial modes

$N \quad-$ Rotational speed

$p, p_{d} \quad-$ Free-field and induct acoustic pressure

$P_{0} \quad-$ Amplitude of free-field acoustic pressure

$R \quad$ - Duct radius

$(r, \varphi, z)$ - Cylindrical coordinates inside the duct

$t \quad-$ Reception time

$U_{\text {hel }} \quad-$ Helical velocity on a rotor blade $\left(U_{\text {hel }}=a M_{\text {hel }}\right)$

$U_{0} \quad-$ Mean flow velocity $\left(U_{0}=a M_{z}\right)$

$\overrightarrow{\mathrm{u}} \quad-$ Acoustic velocity

$v \quad-$ Disturbance velocity normal to a blade

$a-$ Flow angle of attack on the rotor blades

$\beta, \beta_{\theta} \quad-$ Factors of axial Mach number, see Eqs. (4)

$\rho_{0} \quad-$ Fluid mean density

$\sigma \quad-$ Modified distance

$\tau \quad-$ Retarded time

$\Phi \quad-$ Fourier transform of the blade loading

$\Psi \quad-$ Directivity factor of the radiated acoustic field

$\omega \quad-$ Angular frequency $(\omega=2 \pi f=a K)$

\section{Abbreviations}

OAPWL - Overall sound power level

OASPL - Overall sound pressure level

PWL - Sound power level

T\&S - Tyler and Sofrin, see reference 5

\section{INTRODUCTION}

Fan broadband noise often is the major component of overall sound levels radiated by aircraft turbofans at subsonic rotation speeds due to past progress in decreasing the tones. Its prediction has thus become a new challenge for further noise reduction. Forward radiation is mainly due to fluctuating blade pressure (either self-noise, or interaction with incoming turbulence). Computational fluid dynamics (CFD) is not yet able to predict random loads on rotating airfoils, and tests using blade pressure transducers are very expensive. Thus, semiempirical modelling is still being developed. ${ }^{1}$

A prediction method based on analytic equations is a middle way that was proposed in a previous work (see Fig. 1). ${ }^{2}$ It is based on the model of Ffowcs Williams and Hawkings of a random rotating dipole, ${ }^{3}$ and their equation is modified to take into account the Green's function inside a hard-walled infinite cylindrical duct. ${ }^{4}$ Free-field radiation is then calculated using Tyler and Sofrin's (T\&S) model (Rayleigh's integral). ${ }^{5}$ This approach only requires the blade pressure spectrum, assumed to be known, whatever its source may be. An important hypothesis is that there is no reflection on the duct exit, but this is more or less valid except at low frequencies. ${ }^{6}$ Equations were derived for a uniform flow inside the duct and for the free medium at rest (case of static tests). Flow inside the duct was however neglected in the results of reference 2 to balance acoustic powers in the duct and in the free field. Indeed, the hypotheses in the acoustic equations used inside the duct and in the free field were different, due to the flow mismatch at the duct exit.

This paper shows how free-field computation can be extended to a medium in translation which better simulates flight conditions (the same velocity being taken throughout the space). The simple radiation model of T\&S is no longer valid, and a routine based on the full Kirchhoff integral equation is described in the next section. Some results are then discussed for a model fan tested by Rolls-Royce within the framework of the FANPAC (Fan-Noise Prediction and Control) European project. 